What is bridge centrality? A comment on Jones, Ma, and McNally (2019)

\author{
Alexander P. Christensen ${ }^{1}$, Luis Eduardo Garrido², and \& Hudson Golino ${ }^{3}$ \\ ${ }^{1}$ University of Pennsylvania \\ ${ }^{2}$ Pontificia Universidad Católica Madre y Maestra \\ ${ }^{3}$ University of Virginia \\ This preprint has not been peer-reviewed. \\ Read with skepticism. Use the Sagan standard: \\ "extraordinary claims require extraordinary evidence" \\ Author Note
}

Acknowledgements: The authors did not preregister the study. All data, code, and materials can be found on the Open Science Framework: https://osf.io/cza4y/. Alexander P. Christensen (iD https://orcid.org/0000-0002-9798-7037, Luis Eduardo Garrido https://orcid.org/0000-0001-8932-6063, and Hudson Golino iD https://orcid.org/0000-0002-1601-1447.

The authors made the following contributions. Alexander P. Christensen: Conceptualization, Writing - Original Draft Preparation, Writing - Review \& Editing; Luis

Eduardo Garrido: Writing - Review \& Editing; Hudson Golino: Writing - Review \& Editing. Correspondence concerning this article should be addressed to Alexander P. Christensen, Department of Neurology, University of Pennsylvania, Philadelphia, PA, 19104. E-mail: alexpaulchristensen@gmail.com 


\begin{abstract}
Jones, Ma, and McNally (2019) introduce a novel network measure called bridge centrality, which aims to quantify psychopathological symptoms that may contribute to comorbidity. Centrality measures in psychometric networks have come under scrutiny recently with several researchers arguing for more explicit interpretations of what they purport to measure. One useful strategy has been to connect centrality measures to more traditional psychometric measures such as factor loadings. In this commentary, we aim to do the same by connecting bridge centrality to a recently developed measure called network loadings, which are roughly equivalent with factor loadings. By re-analyzing a recent simulation study, we demonstrate that bridge centrality (specifically bridge strength and bridge expected influence) are nothing more than the (absolute) sum of network cross-loadings. We provide an empirical example to demonstrate this equivalency as well as to show some potential shortcomings of bridge centrality when there are more than two disorders in a network. We raise concerns over bridge centrality's utility and recommend researchers use network loadings instead.

Keywords: bridge centrality, cross-loadings, psychometric networks Word count: 3,131 (main text), 166 (abstract)
\end{abstract}




\section{What is bridge centrality? A comment on Jones, Ma, and McNally (2019)}

Psychometric network models have rapidly increased in popularity over the last decade. These models have been used as an alternative perspective to the traditional common cause model suggesting that psychopathological disorders arise from the mutual interactions between symptoms rather than symptoms being caused by the disorder itself (Borsboom \& Cramer, 2013). In these models, nodes (circles) represent symptoms and edges (lines) represent the (conditional) relationships between them. This representation affords a substantive shift for how comorbidity of psychopathological disorders emerge: symptoms that link two disorders together are considered potential "bridges" for one disorder to spread and activate another (Cramer, Waldrop, van der Maas, \& Borsboom, 2010)

Comorbidity of psychopathological disorders is fairly common: as Jones, Ma, and McNally (2019, p. 1) state "if an individual qualifies for one disorder, there is a $45 \%$ chance that he or she qualifies for at least one more" (Kessler, Chiu, Demler, \& Walters, 2005). This makes understanding and treating comorbidity a top priority for clinicians and applied psychologists.

In network models, Blanken et al. (2018) pointed out that investigating symptoms that belong to multiple communities (or factors in network models; Golino \& Epskamp, 2017; Golino et al., 2020) can be used to understand both heterogeneity and comorbidity of psychological disorders. They proposed the use of (overlapping) community detection algorithms to identify stabilizing symptoms (variables with strong within-community connections) and communicating symptoms (variables that belong to multiple communities).

On the one hand, heterogeneity is indicated by symptoms that have strong connections to "problem areas" within a given disorder (i.e., similar to core facets of a higher-order factor). On the other hand, comorbidity is indicated by symptoms that belong to two or more different disorders. To compute the stabilizing values, Blanken et al. (2018) summed the absolute edge weights that a node has within its community while for communicating values the absolute edge weights were summed between communities. 
In the target article, Jones, Ma, and McNally (2019) developed a novel measure called bridge centrality, similar to the "communicating symptoms" idea proposed by Blanken et al. (2018), to quantify the extent to which symptoms in a network act as a "bridge" or connection between two (or more) psychopathological disorders. Bridging symptoms are thought to be relevant for identifying pathways for comorbid disorders and therefore represent potential points of effective intervention.

In light of bridge centrality's popularity (over 70 citations on Google Scholar as of writing), we wondered "what is bridge centrality?" The theoretical motivation is clear but its psychometric meaning is not. Our commentary seeks to provide a definitive answer. For the purposes of this question (and our commentary), we focused solely on undirected networks (edges without arrows). Moreover, we focused on bridge strength and bridge expected influence, which have been the most commonly applied bridge centralities in the literature (e.g., Jones, Ma, \& McNally, 2019; Lazarov et al., 2020).

We organized our commentary by briefly reviewing issues associated with the interpretation of centrality measures, which we argue holds for bridge centralities. We then present a re-analysis of a recent simulation study (Christensen \& Golino, 2021) that demonstrates that bridge centrality is nothing more than the sum of network cross-loadings. We provide an empirical example to show that bridge centrality and network loadings can arrive at the same conclusions. Finally, we end with a discussion on how the applied interpretations of bridge centrality are problematic and warrant caution.

\section{Interpreting Centrality Measures}

Centrality measures are the most commonly applied network measures in the psychometric network literature. Popular measures are betweenness (how often a node used on the shortest path or shortest number of edges from one node to another), closeness (shortest average number of edges to all other nodes), and strength (absolute sum of a node's edge weights). More recently, expected influence (sum of a node's edge weights retaining 
their signs) was introduced (Robinaugh, Millner, \& McNally, 2016). Nodes high on these measures are often interpreted as how "important," "relevant," or "influential" specific symptoms are for psychopathological processes and interventions.

The "influential symptom" interpretation of centrality measures has come under scrutiny in recent years (Bringmann et al., 2019; Hallquist, Wright, \& Molenaar, 2019). Bringmann and colleagues (2019) make several points that are worth repeating: (1) the "influential" interpretation stems from the use of centrality measures in the context of social networks, (2) for all networks, interpretations of centrality measures depend on the data and process being modeled, and (3) meanings about what a "more central" node means should be explicit and match these processes. There have been many attempts to demonstrate that highly central nodes in psychological networks are indeed more "influential" or predictive of changes in psychopathological processes and severity. Unfortunately, these studies have produced mixed results (Dablander \& Hinne, 2019; Elliott, Jones, \& Schmidt, 2020; Rodebaugh et al., 2018; Spiller et al., 2020). Due to their opaque interpretations, Bringmann and colleagues suggested three ways forward: use centrality measures that are more suitable for psychological networks, reconsider them, or abandon them altogether.

Hallquist, Wright, and Molenaar (2019) approached this issue from a traditional psychometric perspective by examining how centrality measures (betweenness, closeness, and strength) are affected by multiple latent causes. In a series of simulations, they demonstrated that betweenness and closeness centrality are strongly affected by sampling variation making their utility for psychological purposes untenable. Strength, however, was demonstrated to be roughly redundant with confirmatory factor analysis (CFA) loadings. Importantly, they discovered that strength reflected an equally weighted combination of independent causes (i.e., factors). This connection to traditional psychometric concepts has been useful for better understanding what is explicitly being measured by strength centrality.

Their findings inspired our own investigation into the extent that strength is equivalent to factor loadings (Christensen \& Golino, 2021). To circumvent the issue of 
strength reflecting a combination of multiple factors, we split strength by these factors and standardized them. These factors in networks (or communities) represent cohesive collections of nodes that are thought to regularly co-occur between people (Christensen, Golino, \& Silvia, 2020). Our formulation for dividing strength by communities is as follows:

$$
\begin{gathered}
S_{i}=\sum_{j=1}^{n}\left|w_{i j}\right|, \\
L_{i f}=\sum_{j \in f}^{F}\left|w_{i j}\right|,
\end{gathered}
$$

where $\left|w_{i j}\right|$ is the absolute weight (e.g., partial correlation) between node $i$ and $j, S_{i}$ is the sum of the edge weights connected to node $i$ across all nodes $(n$; i.e., strength for node i), $L_{i f}$ is the sum of edge weights in community $f$ that are connected to node $i$ (i.e., node $i$ 's strength for community $f$ ), and $F$ is the number of communities (in the network). This measure can be standardized using the following formula:

$$
z_{L_{i f}}=\frac{L_{i f}}{\sqrt{\sum L_{. f}}},
$$

where the denominator is equal to the square root of the sum of all the weights in community $f$. Notably, the standardized loadings are absolute weights with the signs being added after the loadings have been computed (following the same procedure as factor loadings; Comrey \& Lee, 2013).

We called this formulation network loadings and evaluated their equivalency to factor loadings across three simulations. These simulations demonstrated that network loadings successfully circumvent the issue of multiple latent causes and that they are roughly equivalent to exploratory factor analysis (EFA) loadings. Importantly, we were able to develop guidelines for network loading effect sizes: small (0.15), moderate (0.25), and large $(0.35)$.

Our simulations demonstrated that network loadings have an explicit interpretation: 
how well a variable measures a latent factor. We suggested that from a network perspective this interpretation shifts to: each variable's (unique) contribution to the emergence of a coherent dimension (or collection of related variables) in a network. Our study, in combination with Hallquist, Wright, and Molenaar (2019), sets a precedent for understanding what node strength is: the unstandardized sum of the absolute dominant and cross-loadings. We believe that such a precedent should be set for any new measures introduced into the psychometric network literature.

\section{What is Bridge Centrality?}

From a substantive perspective, the answer is clear: symptoms high in bridge centrality may contribute to psychopathological comorbidity and intervening on these symptoms may mitigate comorbidity (Jones, Ma, \& McNally, 2019). Statistically speaking, bridge centralities are computed similarly to network loadings: using communities and computing a centrality measure. For example, bridge strength can be computed by taking the absolute sum of a node's strength to communities other than it's own (i.e., inter-community but not intra-community edges or what Blanken et al. (2018) called "communicating symptoms"). This is defined as:

$$
\text { bridge strength }=\sum_{b \in(N(a)-C)}\left|w_{a b}\right| .
$$

Consider that this expression is mathematically equivalent to the unstandardized network

loadings above $\left(L_{i f}\right)$ but summing across all communities except for the community that the node belongs to.

Similarly, bridge expected influence is computed the same way and only differs in that signed values of edges $\left(w_{a b}\right)$ are used rather than absolute values $\left(\left|w_{a b}\right|\right)$. Importantly, Jones, Ma, and McNally (2019) demonstrate that these measures go above and beyond traditional centrality statistics for reducing comorbidity in their simulations.

But what do these measures mean psychometrically? Based on their mathematical 
definitions, bridge strength and bridge expected influence are closely related to network loadings where bridge strength is the absolute sum of edge weights that do not belong to the node's community. Bridge expected influence is the signed sum of these edge weights. In more traditional terms, bridge strength and bridge expected influence are equivalent to the (absolute) sum of the network cross-loadings.

To demonstrate this equivalence, we re-analyzed our recent simulation which compared factor analysis loadings with network loadings (Christensen \& Golino, 2021). In our simulation, we generated data from a multivariate normal factor models with two, three, and four factors with cross-loadings. We generated both continuous and polytomous data (i.e., 5-point Likert scale). For more details on the simulation conditions, see Christensen and Golino (2021).

To compare the bridge centralities and network loadings, we used the simulated factor structure to designate the communities. This allowed for direct comparisons that were not affected by dimension estimation methods (e.g., exploratory graph analysis; Golino \& Epskamp, 2017; Golino et al., 2020). We then summed the absolute and signed values of the network cross-loadings to mirror bridge strength and bridge expected influence, respectively.

Bridge expected influence can be computed with one step (which is equivalent to signed strength) and two steps. The only difference for expected influence with two steps is that a node's neighbors' connections are also included in the sum - that is, it's the sum of a node's connections and its neighbors' connections. We used Spearman's rank-order correlations which take into account whether the nodal metrics are in the same order (rather than whether there was a linear effect).

Our continuous data results confirmed our expectations: bridge strength was strongly correlated with the absolute sum of the network cross-loadings $\left(\bar{r}=.977, r_{\text {median }}=0.996\right.$, $\left.r_{S D}=0.057\right)$. Similarly, both bridge expected influence 1 -step $\left(\bar{r}=.987, r_{\text {median }}=0.997\right.$, $\left.r_{S D}=0.035\right)$ and 2-step $\left(\bar{r}=.939, r_{\text {median }}=0.971, r_{S D}=0.084\right)$ were strongly correlated with the sum of the network cross-loadings. Our polytomous data results followed the same 
pattern: bridge strength $\left(\bar{r}=.959, r_{\text {median }}=0.992, r_{S D}=0.082\right)$, bridge expected influence 1-step $\left(\bar{r}=.961, r_{\text {median }}=0.990, r_{S D}=0.080\right)$, and 2-step $\left(\bar{r}=.926, r_{\text {median }}=0.957\right.$, $\left.r_{S D}=0.091\right)$. In short, our results support the interpretation that bridge centrality is the (absolute) sum of network cross-loadings.

\section{Empirical Example}

Jones, Ma, and McNally (2019) applied bridge strength to several different empirical examples in the literature that examined the comorbidity of psychopathological disorders. For many of their examples (and we suspect for much of the literature) comorbidity was examined with two disorders. In these instances, bridge strength and network cross-loadings will be equal. When there are more than two disorders (or dimensions), then the sum of network cross-loadings will be equal to bridge centrality.

To demonstrate this latter equivalency empirically, we used the bulimia nervosa, anxiety, and depression network in Jones, Ma, and McNally's (2019) supplementary information that was originally published by Levinson et al. (2017). In their original analysis, Jones, Ma, and McNally (2019) computed bridge strength for the network of these three disorders and reported the symptoms that were in the 80 percentile or higher on bridge strength (Table 3 of Jones, Ma, \& McNally, 2019). We replicated this example by computing the absolute network loadings and summing across the cross-loadings.

Similarly, we report the symptoms that were in the 80 percentile or higher here (names in parentheses correspond to node names in Table 1 and Figure 1): concentration problems (concentration), crying (crying), difficulty making decisions (indicisive), discomfort with exposure (expodiscom), disliking self (selfdislike), fear of the worst happening (fearofworst), feeling agitated and restless (agitation), feeling nervous (nervous), numbness or tingling (tingling), pessimism, sadness (sadness), self-criticalnness (critical), sleep problems (sleep), terrified (terrified), and unable to relax (unabletorelax).

Our results fully replicate Jones, Ma, and McNally's (2019) findings with one 
additional symptom, pessimism, that was found. Pessimism was exactly at the 80 percentile cut-off for network loadings. Computing Spearman's rank-order correlation between all variables' bridge strength and absolute sum of the network cross-loadings, we found that they were perfectly related $(r=0.999)$.

Importantly, network loadings capture information that bridge strength does not: The disorders that these bridge symptoms actually bridge. For example, the feeling agitated symptom of depression is a bridge but is it a bridge to bulimia nervosa, anxiety, or both? Because bridge centralities collapse across communities, there is no way to answer this question with certainty. Below, however, we show that network loadings can provide insight to this question.

Table 1

Network Loadings for the High Bridge Strength Symptoms

\begin{tabular}{lccc}
\hline & Bulimia Nervosa & Anxiety & Depression \\
\hline agitation & 0.002 & 0.061 & 0.146 \\
concentration & 0.021 & 0.026 & 0.225 \\
critical & 0.027 & 0.018 & 0.199 \\
crying & 0.033 & 0.015 & 0.138 \\
expodiscom & 0.182 & 0.000 & 0.040 \\
fearofworst & 0.000 & 0.164 & 0.047 \\
indiscisive & 0.008 & 0.031 & 0.161 \\
nervous & 0.004 & 0.162 & 0.046 \\
sadness & 0.006 & 0.030 & 0.281 \\
selfdislike & 0.048 & 0.000 & 0.181 \\
sleep & -0.013 & 0.025 & 0.102 \\
terrified & 0.015 & 0.182 & 0.020 \\
tingling & 0.000 & 0.090 & 0.040 \\
unabletorelax & 0.000 & 0.124 & 0.057 \\
\hline
\end{tabular}

From Table 1, we can see a more nuanced breakdown of which symptoms "bridge" to other disorders. The feeling agitated (agitation) symptom of depression has a larger cross-loading for anxiety (0.061) than for bulimia nervosa (0.002). If we were interested in symptoms that are bridges to bulimia nervosa, then feeling agitated wouldn't be worthy of much consideration. Using bridge centrality, however, we might be tempted to draw that 
conclusion. Symptoms such as discomfort with exposure (expodiscom), crying (crying), and disliking self (selfdislike) would be of more interest. Interestingly, all three of these symptoms are associated with depression and less so anxiety. In fact, there does not appear to be any symptoms that are "high" in bridge centrality between bulimia nervosa and anxiety.

Comorbidity studies are likely to benefit when more than two psychopathological disorders are included. Because psychometric networks condition symptoms across all others, there is less likely to be a third variable problem when multiple disorders are used. In our empirical example, we could erroneously conclude that there are several symptoms that bridge between bulimia nervosa and anxiety if these are the only two disorders included in the network (Jones, Ma, \& McNally, 2019; Levinson et al., 2017). When depression is included with bulimia nervosa and anxiety, then we would instead conclude that depression symptoms bridge bulimia nervosa symptoms to anxiety symptoms. That is, there are more (and stronger) associations between bulimia nervosa and depression symptoms than bulimia nervosa and anxiety symptoms (Table 1). Indeed, this is apparent in the visual representation of the three disorders where there are fewer edges going between bulimia nervosa and anxiety (22) than bulimia nervosa and depression (38; Figure 1). 


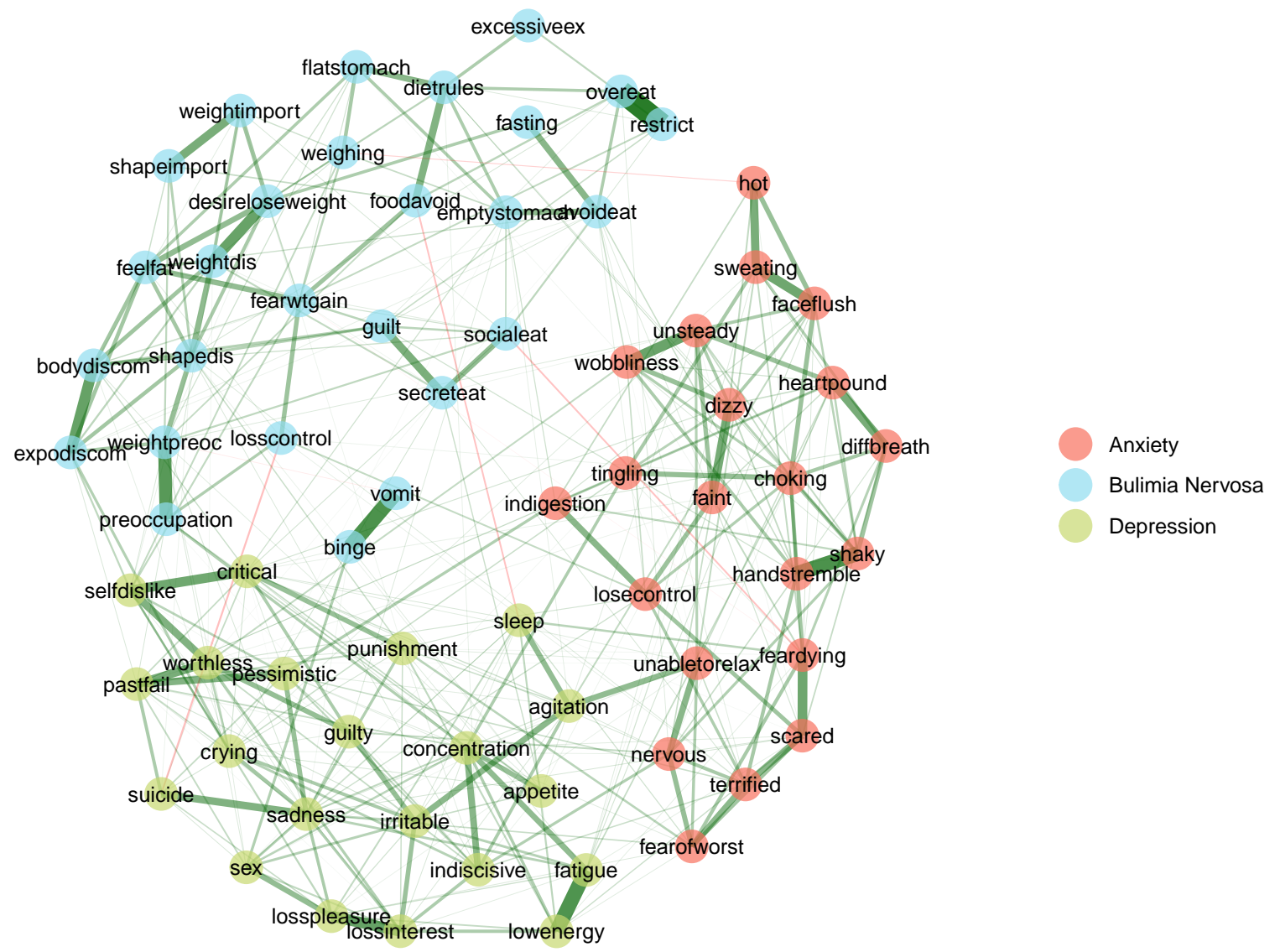

Figure 1

Network of Bulimia Nervosa, Depression, and Anxiety

\section{Narrow Bridge Ahead}

In our commentary on Jones, Ma, and McNally (2019), we demonstrated that bridge centrality is equal to the sum of the network cross-loadings. Such an explanation provides an explicit meaning to what bridge centrality is to researchers using the measure. Importantly, we demonstrated that bridge centrality misses useful information when there are more than two psychopathological disorders, potentially leading to erroneous conclusions and raising the issue of its utility for the study of comorbidity in these conditions.

Even in conditions where there are only two psychopathological disorders, we urge caution when applying bridge centrality. Bridge centrality, like more traditional centrality measures, is relative which means that effects across studies are difficult to compare. 
Moreover, bridge centrality has no indication of effect size making conclusions about what bridge symptoms are meaningful subjective at best (e.g., 80 percentile cut-off). We argue that these constraints of bridge centrality make its utility far too narrow for widespread use.

Instead, we recommend using network loadings because they offer several benefits over bridge centrality. First, their effects are standardized making comparisons between samples much more direct. Second, network loadings have effect sizes that have been validated via simulation (Christensen \& Golino, 2021). Network cross-loadings will tend to be small (and usually smaller than a small effect size), but when there are cross-loading effects that achieve a magnitude of at least .10, then they may be considered substantively meaningful. Finally, network loadings provide information about which symptoms are "bridging" to which disorders (or communicating to other disorders; Blanken et al., 2018).

Given the equivalence of factor and network loadings, we can give meaning to bridge centrality from a factor analytic perspective: bridge centrality is the aggregated latent effect of non-dominant causes (cross-loadings) on a variable. Cross-loadings have largely been ignored in modern psychometrics and are considered to be problematic for valid measurement (DeVellis, 2017; Furr, 2017).

Because researchers who employ factor analysis are principally concerned with the interpretation of latent constructs and their relations, they have typically viewed cross-loadings as a nuisance (Little, Rhemtulla, Gibson, \& Schoemann, 2013). Items with substantial cross-loadings often have lower primary loadings, resulting in less stable structures and lower construct reliability (Costello \& Osborne, 2005; Li, Wen, Hau, Yuan, \& Peng, 2020). Additionally, factors composed of such items are more difficult to interpret due to the multidimensionality of their indicators and to diminished discriminant validities (Arias, Ponce, Martínez-Molina, Arias, \& Núñez, 2016; Farrell, 2010; Li, Wen, Hau, Yuan, \& Peng, 2020). Although some amount of cross-loading variance is unavoidable due to the fallible nature of psychological indicators (Arias, Ponce, Martínez-Molina, Arias, \& Núñez, 2016; Morin, Arens, \& Marsh, 2016), researchers generally try to limit their impact by removing 
items that exhibit many or high cross-loadings from inventories (Costello \& Osborne, 2005). Even though the mechanisms posited by network models differ from those of factor analysis, the factor analytic literature provides information that suggests caution when interpreting bridge centrality. On the one hand, it is known that cross-loadings can arise due a wide array of causes, which are difficult to disentangle. For example, non-substantive causes such as poorly written items, method effects, item wording similarity, and other response biases, can lead to cross-loadings (Costello \& Osborne, 2005; Marsh, Lüdtke, Nagengast, Morin, \& Von Davier, 2013; Morin, Arens, \& Marsh, 2016). Additionally, cross-loadings can arise from alternative substantive causes such as the presence of an underlying general factor (Arias, Ponce, Martínez-Molina, Arias, \& Núñez, 2016; Morin, Arens, \& Marsh, 2016). Often cross-loadings imply variance that is specific to the item, interpreting its meaning becomes difficult given the potential wide array of factors (substantive and non-substantive) that can influence them. On the other hand, cross-loadings are typically small loadings that have large standard errors and less stability across samples (Sass, 2010). As a result, researchers may need to collect prohibitively large samples to be able to determine with sufficient precision the values of cross-loadings or bridges between specific pairs of disorders.

In sum, we argue that bridge centrality has limited utility from a psychometric perspective. It lacks standards to make meaningful between sample comparisons and nuances to determine which symptoms bridge to other disorders. We urge caution with the psychometric information being considered by bridge centrality; instead, we recommend the use of network loadings which provide more complete and explicit psychometric information. 


\section{References}

Arias, V. B., Ponce, F. P., Martínez-Molina, A., Arias, B., \& Núñez, D. (2016). General and specific attention-deficit/hyperactivity disorder factors of children 4 to 6 years of age: An exploratory structural equation modeling approach to assessing symptom multidimensionality. Journal of Abnormal Psychology, 125, 125. https://doi.org/10.1037/abn0000115

Blanken, T. F., Deserno, M. K., Dalege, J., Borsboom, D., Blanken, P., Kerkhof, G. A., \& Cramer, A. O. J. (2018). The role of stabilizing and communicating symptoms given overlapping communities in psychopathology networks. Scientific Reports, 8, 5854 . https://doi.org/10.1038/s41598-018-24224-2

Borsboom, D., \& Cramer, A. O. J. (2013). Network analysis: An integrative approach to the structure of psychopathology. Annual Review of Clinical Psychology, 9, 91-121. https://doi.org/10.1146/annurev-clinpsy-050212-185608

Bringmann, L. F., Elmer, T., Epskamp, S., Krause, R. W., Schoch, D., Wichers, M., ... Snippe, E. (2019). What do centrality measures measure in psychology networks? Journal of Abnormal Psychology, 128, 892-903. https://doi.org/10.1037/abn0000446

Christensen, A. P., \& Golino, H. (2021). On the equivalency of factor and network loadings. Behavior Research Methods. https://doi.org/10.3758/s13428-020-01500-6

Christensen, A. P., Golino, H., \& Silvia, P. J. (2020). A psychometric network perspective on the validity and validation of personality trait questionnaires. European Journal of Personality, 34, 1095-1108. https://doi.org/10.1002/per.2265

Comrey, A. L., \& Lee, H. B. (2013). A first course in factor analysis (2nd ed.). New York, NY: Psychology Press.

Costello, A. B., \& Osborne, J. (2005). Best practices in exploratory factor analysis: Four recommendations for getting the most from your analysis. Practical Assessment, Research, and Evaluation, 10, 7. https://doi.org/10.7275/jyj1-4868

Cramer, A. O. J., Waldrop, L. J., van der Maas, H. L., \& Borsboom, D. (2010). 
Comorbidity: A network perspective. Behavioral and Brain Sciences, 33, 137-150. https://doi.org/10.1017/S0140525X09991567

Dablander, F., \& Hinne, M. (2019). Node centrality measures are a poor substitute for causal inference. Scientific Reports, 9, 6846. https://doi.org/10.1038/s41598-019-43033-9

DeVellis, R. F. (2017). Scale development: Theory and applications (4th ed.). Thousand Oaks, CA: SAGE Publications.

Elliott, H., Jones, P. J., \& Schmidt, U. (2020). Central symptoms predict posttreatment outcomes and clinical impairment in anorexia nervosa: A network analysis. Clinical Psychological Science, 8, 139-154. https://doi.org/10.1177/2167702619865958

Farrell, A. M. (2010). Insufficient discriminant validity: A comment on Bove, Pervan, Beatty, and Shiu (2009). Journal of Business Research, 63, 324-327. https://doi.org/10.1016/j.jbusres.2009.05.003

Furr, M. R. (2017). Psychometrics: An introduction (3rd ed.). Thousand Oaks, CA: SAGE Publications.

Golino, H., \& Epskamp, S. (2017). Exploratory Graph Analysis: A new approach for estimating the number of dimensions in psychological research. PloS ONE, 12, e0174035. https://doi.org/10.1371/journal.pone.0174035

Golino, H., Shi, D., Christensen, A. P., Garrido, L. E., Nieto, M. D., Sadana, R., ... Martinez-Molina, A. (2020). Investigating the performance of Exploratory Graph Analysis and traditional techniques to identify the number of latent factors: A simulation and tutorial. Psychological Methods, 25, 292-320. https://doi.org/10.1037/met0000255

Hallquist, M., Wright, A. C. G., \& Molenaar, P. C. M. (2019). Problems with centrality measures in psychopathology symptom networks: Why network psychometrics cannot escape psychometric theory. Multivariate Behavioral Research. https://doi.org/10.1080/00273171.2019.1640103

Jones, P. J., Ma, R., \& McNally, R. J. (2019). Bridge centrality: A network approach to understanding comorbidity. Multivariate Behavioral Research, 1-15. 
https://doi.org/10.1080/00273171.2019.1614898

Kessler, R. C., Chiu, W. T., Demler, O., \& Walters, E. E. (2005). Prevalence, severity, and comorbidity of 12-month DSM-IV disorders in the National Comorbidity Survey Replication. Archives of General Psychiatry, 62, 617-627. https://doi.org/10.1001/archpsyc.62.6.617

Lazarov, A., Suarez-Jimenez, B., Levi, O., Coppersmith, D. D., Lubin, G., Pine, D. S., ... Neria, Y. (2020). Symptom structure of PTSD and co-morbid depressive symptoms - a network analysis of combat veteran patients. Psychological Medicine, 50, 2154-2170. https://doi.org/10.1017/S0033291719002034

Levinson, C. A., Zerwas, S., Calebs, B., Forbush, K., Kordy, H., Watson, H., ... Bulik, C. M. (2017). The core symptoms of bulimia nervosa, anxiety, and depression: A network analysis. Journal of Abnormal Psychology, 126, 340-354. https://doi.org/10.1037/abn0000254

Li, Y., Wen, Z., Hau, K.-T., Yuan, K.-H., \& Peng, Y. (2020). Effects of cross-loadings on determining the number of factors to retain. Structural Equation Modeling: A Multidisciplinary Journal, 1-23. https://doi.org/10.1080/10705511.2020.1745075

Little, T. D., Rhemtulla, M., Gibson, K., \& Schoemann, A. M. (2013). Why the items versus parcels controversy needn't be one. Psychological Methods, 18, 285-300. https://doi.org/10.1037/a0033266

Marsh, H. W., Lüdtke, O., Nagengast, B., Morin, A. J. S., \& Von Davier, M. (2013). Why item parcels are (almost) never appropriate: Two wrongs do not make a right - Camouflaging misspecification with item parcels in CFA models. Psychological Methods, 18, 257-284. https://doi.org/10.1037/a0032773

Morin, A. J. S., Arens, A. K., \& Marsh, H. W. (2016). A bifactor exploratory structural equation modeling framework for the identification of distinct sources of construct-relevant psychometric multidimensionality. Structural Equation Modeling: A Multidisciplinary Journal, 23, 116-139. https://doi.org/10.1080/10705511.2014.961800 
Robinaugh, D. J., Millner, A. J., \& McNally, R. J. (2016). Identifying highly influential nodes in the complicated grief network. Journal of Abnormal Psychology, 125, 747-757. https://doi.org/10.1037/abn0000181

Rodebaugh, T. L., Tonge, N. A., Piccirillo, M. L., Fried, E., Horenstein, A., Morrison, A. S., ... Heimberg, R. G. (2018). Does centrality in a cross-sectional network suggest intervention targets for social anxiety disorder? Journal of Consulting and Clinical Psychology, 86, 831-844. https://doi.org/10.1037/ccp0000336

Sass, D. A. (2010). Factor loading estimation error and stability using exploratory factor analysis. Educational and Psychological Measurement, 70, 557-577. https://doi.org/10.1177/0013164409355695

Spiller, T. R., Levi, O., Neria, Y., Suarez-Jimenez, B., Bar-Haim, Y., \& Lazarov, A. (2020). On the validity of the centrality hypothesis in cross-sectional between-subject networks of psychopathology. BMC Medicine, 18, 1-14. https://doi.org/10.1186/s12916-020-01740-5 